\title{
The Numerical Modeling and Measurement of Demagnetization Effect in Bulk YBCO Superconductors Subjected to Transverse Field
}

\author{
Z. Hong, Ph. Vanderbemden, R. Pei, Y. Jiang, A. M. Campbell, and T. A. Coombs
}

\begin{abstract}
In this paper, the crossed field demagnetization effect of the bulk type-II superconducting materials in a variety of external magnetic field condition has been investigated both numerically and experimentally. A melt-textured YBCO bulk sample was magnetized along the axial direction and after that a transverse field perpendicular to the central axis applied. The collapse of the original trapped field was measured using a Quantum Design Physical Property Measurement System. A numerical solver based on critical state model is proposed to simulate the measured results.
\end{abstract}

Index Terms-Bulk superconductor, critical state model, crossed field effect, finite element method, numerical modeling.

\section{INTRODUCTION}

$\mathbf{T}$ HE potential of bulk melt-processed YBCO single domains to trap significant magnetic field at $77 \mathrm{~K}$ makes them particularly attractive for a variety of engineering applications including superconducting magnets, magnetic bearings and motors [1], [2]. Before such devices can be created, the characteristics of these trapped field magnets need to be well understood. A major cause of concern is the phenomenon of magnetization decay, which has been observed when the material is subjected to magnetic fields in a direction transverse to the original magnetization, result in magnets losing their magnetization and thus becoming worthless in applications. In spite of having been investigated for more than three decades, the subject remains a very intriguing topic for which many experimental facts are still far from being understood. In particular, we need to know whether large numbers of cycles of the crossed field lead eventually to total demagnetization, as suggested by some experiments and theories [3], or the decay of the magnetization ceases at a useful value as predicted by theories based on the critical state model [4], [5]. In this paper, a melt-textured YBCO bulk sample was magnetized along the axial direction and after that a transverse field perpendicular to the central axis applied. The collapse of the original trapped field was measured using a Quantum Design Physical Property

Manuscript received August 22, 2007

Z. Hong, R. Pei, Y. Jiang, A. M. Campbell, and T. A. Coombs are with the Engineering Department, Cambridge University, Cambridge CB2 1PZ, U.K. (e-mail: zh223@cam.ac.uk).

P. Vanderbemden is with SUPRATECS, Department of Electrical Engineering and Computer Science B28, University of Liège, Sart-Tilman, B-4000 Liège, Belgium.

Color versions of one or more of the figures in this paper are available online at http://ieeexplore.iee.org.

Digital Object Identifier 10.1109/TASC.2008.920594
Measurement System (PPMS) and found that the decay of the magnetization can be fitted using a power law $M \sim N^{\alpha}$ where $\mathrm{N}$ is the number of the transverse field sweeps. A two dimensional numerical model based on a set of Maxwell's equations is proposed to simulate the electromagnetic behavior of the YBCO bulks. The model assumes a field dependent $J_{\mathrm{c}}$ and is used with $E-J$ power constitutive law; it can qualitatively reproduce the experimental results and help to investigate the current and field distributions throughout the cross sectional area of the sample.

\section{NUMERICAL MODEL}

The numerical solver of the critical state used in this paper is based on a set of Maxwell's equations using magnetic field $H_{x}$ and $H_{y}$ as the knowns. The equations are incorporated with a commercial finite element software Comsol Multiphysics.

According to Faraday's Law,

$$
\nabla \times \mathbf{E}=-\mu_{0} \mu_{r} \frac{\partial \mathbf{H}}{\partial t}
$$

The magnetic flux is flowing in the $x-y$ plane and the magnetic field is used as the dependent variable $\mathbf{H}=\left[H_{x} H_{y}\right]$ representing the components of the magnetic field in the $\mathrm{x}$ and $\mathrm{y}$ directions. The induced electric field and the current density only have the components in the z-direction. So $E=E_{s c_{-} z}$ and $J=J_{s c_{-} z}$. The electrical behavior of the superconducting material is represented by the $E-J$ power law. In this case, it is expressed as:

$$
E_{s c_{-} z}=E_{0}\left(\frac{J_{s c_{-} z}}{J_{c}(B)}\right)^{n}
$$

where $J_{c}$ is the critical current density (defined with the standard $1 \mu \mathrm{V} / \mathrm{cm}$ electric field criterion). $E_{0}=1 \times 10^{-4} \mathrm{~V} / \mathrm{m}$ and the value of " $n$ " is determined by the characteristics of the superconducting materials.

According to Ampere's law:

$$
\nabla \times H=J
$$

Since $J_{s c_{-} z}$ only has the component in the z-direction, Ampere's law in this case is expressed as:

$$
J_{s c_{-z}}=\frac{\partial H_{y}}{\partial x}-\frac{\partial H_{x}}{\partial y}
$$




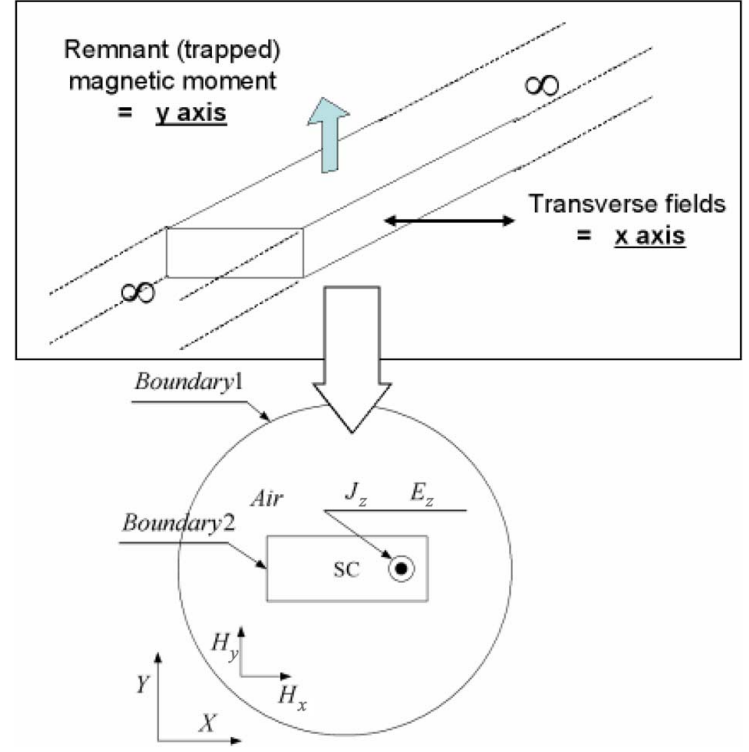

Fig. 1. Diagram of model, subdomains and boundaries. The magnetic field is in the x-y plane and the critical current density $J_{z}$ and the induced electric field $\mathrm{E}_{\mathrm{z}}$ are in the $\mathrm{z}$-direction.

Substitution of (2) and (4) into (1) gives two coupled equations:

$$
\begin{array}{r}
\partial\left(E_{0}\left(\frac{\frac{\partial H_{y}}{\partial x}-\frac{\partial H_{x}}{\partial y}}{J_{c}(B)}\right)^{n}\right) / \partial y=-\mu_{0} \mu_{r} \frac{\partial H_{x}}{\partial t} \\
-\partial\left(E_{0}\left(\frac{\frac{\partial H_{y}}{\partial x}-\frac{\partial H_{x}}{\partial y}}{J_{c}(B)}\right)^{n}\right) / \partial x=-\mu_{0} \mu_{r} \frac{\partial H_{y}}{\partial t}
\end{array}
$$

Equations (5) and (6) include two PDEs and two scalar dependent variables $H_{x}$ and $H_{y}$. Combining this with suitable boundary conditions, they can be solved with FEM software.

On the outer boundary of the non-superconducting region (boundary 1 in Fig. 1), a Dirichlet boundary condition can be set up as:

$$
\left\{\begin{array}{l}
H_{x}=f_{x}(t) \\
H_{y}=f_{y}(t)
\end{array}\right.
$$

Where $f_{x}(t)$ and $f_{y}(t)$ are functions that describes how the external field in the $\mathrm{x}$ and $\mathrm{y}$ directions vary with time.

\section{EXPERIMENT}

Small sample of bulk melt-processed single domain YBCO with an aspect ratio of $\sim 1: 3$ (typical size $0.8 \times 0.8 \times 0.25 \mathrm{~mm}$ ) were cut from the parent single domain using a wire saw. These samples were characterized magnetically for applied field conditions of $H \| \mathrm{ab}$ and $H \| \mathrm{c}$ at $\mathrm{T}=77 \mathrm{~K}$ using a Quantum Design SQUID Magnetic Property Measurement System (MPMS). The full penetration fields along $a b$ plane and $c$ axis are $64 \mathrm{mT}$ and $74 \mathrm{mT}$ respectively.

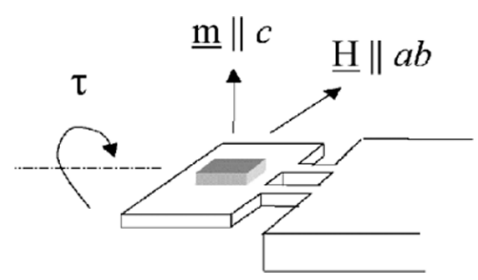

Fig. 2. Schematic illustration of the sample mounted on the platform used for torque measurements.

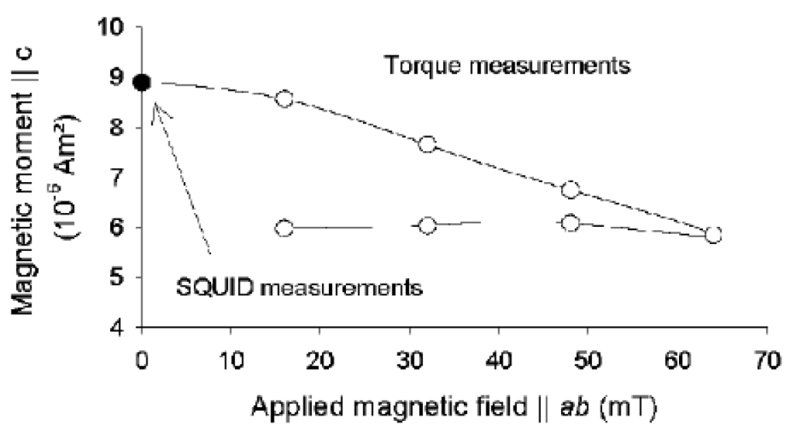

Fig. 3. Magnetic moment $\| c$-axis during the application of one sweep of transverse field $\| a b$. Black symbol: initial trapped magnetic moment without transverse field. White symbols: data determined from magnetic torque measurements.

Measurements of the YBCO samples in the crossed-field configuration were carried out using magnetic torque magnetometry in PPMS. In this case, the magnetic torque can be expressed as;

$$
\underline{\tau}=\underline{m}_{c} \times \mu_{0} \underline{H}_{a b}
$$

The variation of magnetic moment $\mathrm{m}_{\mathrm{c}}$ vs. the applied field $\mathrm{H}_{\mathrm{ab}}$ can be determined from the measured torque by $\mathrm{m}_{\mathrm{c}}\left(H_{\mathrm{ab}}\right)=\tau\left(H_{\mathrm{ab}}\right) /\left(\mu_{0} H_{\mathrm{ab}}\right)$, since $\mathrm{m}_{\mathrm{c}}$ and $H_{\mathrm{ab}}$ are mutually orthogonal, as shown in Fig. 2. This technique is particularly convenient for determining accurately the magnetic moment of small-size superconductors subjected to crossed fields.

However, one constraint of the method is that the measurements must always be performed in the presence of a small but finite transverse field $H_{\mathrm{ab}}$. As shown in Fig. 3, the measured $\mathrm{m}_{\mathrm{c}}\left(H_{\mathrm{ab}}\right)$ data obtained by torque measurements agree perfectly with the initial value of magnetic moment determined by SQUID magnetometry in the absence of a transverse field.

In all experiments, the melt-processed sample was initially magnetized parallel to the $c$-axis by field cooling (FC) down to $\mathrm{T}=77 \mathrm{~K}$ in an applied magnetic flux density of $0.5 \mathrm{~T}$. The field amplitude was intentionally much larger than the full-penetration field of the sample $\left(\mu_{0} H_{\mathrm{p}}=64 \mathrm{mT}\right)$ to ensure that the maximum (trapped) magnetic moment $\mathrm{m}_{\mathrm{c}}$ is achieved [6]. A constant time interval of approximately 5 minutes was allowed for magnetic relaxation following the removal of the applied ( $c$-axis) field [7]. A transverse magnetic field $H_{\mathrm{ab}}$ was then applied parallel to the $a b$ plane of the sample and the magnetic 


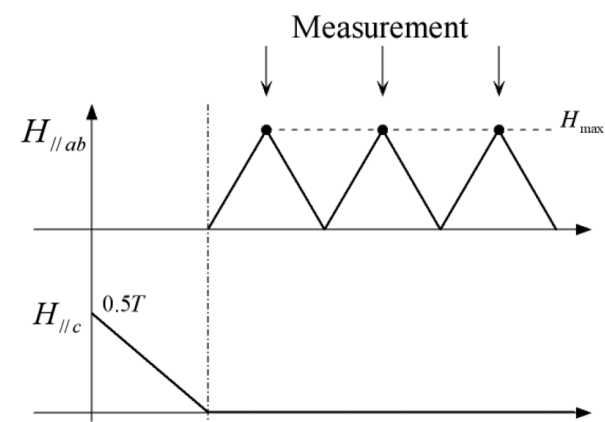

Fig. 4. Schematic illustration and the sequences of magnetic fields applied to the sample.

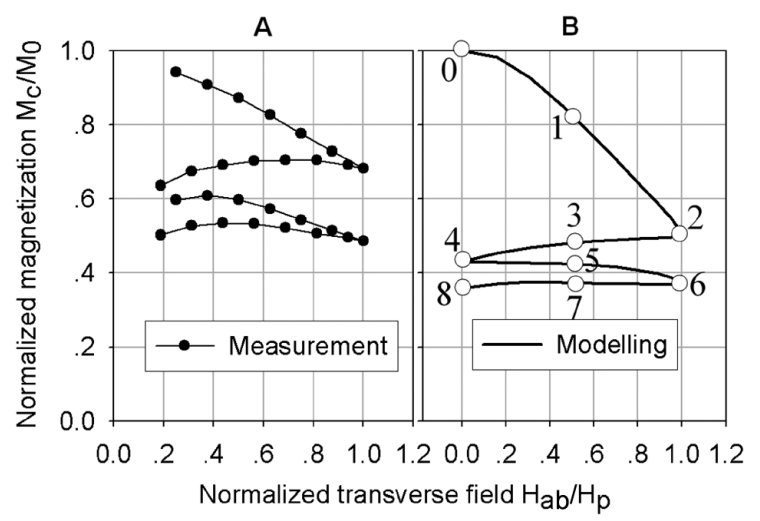

Fig. 5. Measured and modeled magnetization $\| \mathrm{c}$ during the application of two cycles of transverse field $\| \mathrm{ab}$. The magnetization is normalized with respect to its initial value $M_{0}$. The transverse field is normalized with respect to the full penetration field $H_{p}$.

torque recorded, as illustrated schematically in Fig. 2 and Fig. 4.

\section{RESULTS}

\section{A. Demagnetization in the First and Second Cycle}

Fig. 5(a) shows the influence of transverse magnetic field sweeps $H_{\mathrm{ab}}$ on the trapped magnetization $M_{\mathrm{c}}$ (\| c-axis) measured by a $P P M S$. The magnetization is normalized with respect to its initial value, $M_{0}$, measured by SQUID, whereas the transverse field is normalized with respect to the full-penetration field $H_{\mathrm{p}}$ in the $a b$ direction. The experimental data display the remaining $M_{\mathrm{c}}$ after one cycle is found to be $\sim 0.62 M_{0}$. Secondly, the consistent decreases in $M_{\mathrm{c}}$ observed when $H_{\mathrm{ab}}$ is swept from 0 to $+H_{\max }$ are generally much larger than the decrease in magnetization caused by sweeps from $+H_{\max }$ to 0 . Thirdly, the results displayed in Fig. 5(a) also show that the second cycle of the transverse field causes less magnetization decrease than that of the first cycle

Fig. 5(b) shows the results of the modeling, corresponding to the experimental conditions described above. From a quantitative point of view, the induction decays resulting from one complete oscillation of the transverse field are found to be much more pronounced than those measured experimentally: after one cycle of $H_{\mathrm{ab}}$, the remaining $M_{\mathrm{c}}$ is only $\sim 0.45 M_{0}$, compared to $\sim 0.62 B_{0}$ for the experimental data. These differences are due to the differences between the model and experimental geometries. From a qualitative point of view, however, the modeled

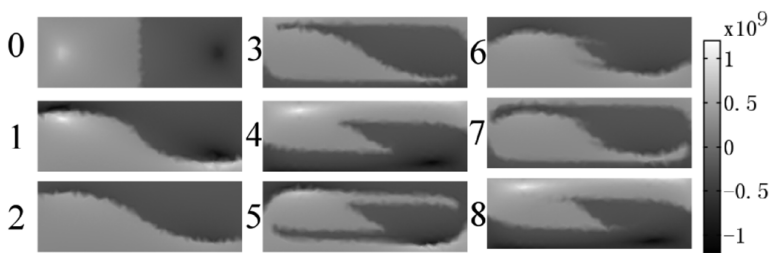

Fig. 6. Modeled data of the current density distribution $J_{z_{-s c}}(\mathrm{x}, \mathrm{y})$ within the cross-section of the sample during one cycle of the transverse magnetic field of amplitude $0.5 H_{\mathrm{p}}$. The number of images correspond to the time steps listed in Fig. 5(b).

$M_{\mathrm{c}}\left(H_{\mathrm{ab}}\right)$ curves are remarkably similar to the experimental data. In particular, the $M_{\mathrm{c}}\left(H_{\mathrm{ab}}\right)$ segments that correspond to sweeps from $+H_{\max }$ to 0 and from $-H_{\max }$ to 0 are nearly horizontal, showing clearly that the remanent magnetization is affected only weakly by the transverse field in this regime.

Fig. 6 shows the current distribution modeled within the sample cross-section at several selected times during the first and the second transverse field cycle for transverse field amplitude of $H_{\mathrm{p}}$. The images correspond to the time steps listed in Fig. 5(b). The surface plot represents the current density; the light region stands for the positive current and the dark region stands for the negative current. Image 0 shows the current distribution that gives rise to a positive trapped magnetization (i.e. $M_{\mathrm{c}}>0$ ). The magnitude of the current density is not uniform. This arises from the $J_{\mathrm{c}}(B)$ dependence. The application of a positive transverse field $H_{\mathrm{ab}}>0$ leads to a reversal of the current density in the top-left and bottom-right regions of the sample, as shown in Image 1. The consequence is that both upper and lower layers of the sample no longer contribute efficiently to the c-axis magnetization $M_{\mathrm{c}}$. Increasing the field (Image 2) increases the distortion of the current distribution. On lowering the field (Images 3 and 4), the shielding currents in the top and bottom layers change their sign, as required for shielding the decreasing applied field $\mathrm{d} H_{\mathrm{ab}} / \mathrm{dt}<0$. However the current distribution in the central horizontal layer of the sample remains unaffected. The corresponding decay of $\mathrm{M}_{\mathrm{c}}$ is therefore very small, as observed in Fig. 5.

\section{B. Large Number of Sweeps of Transverse Field}

After the sample is magnetized by field cooling, a transverse field demagnetization sequence is cycled by 120 times between 0 and $+H_{\max }$. This measurement is taken with $H_{\max }$ equals to 0.25 and 0.5 full penetration field $\left(H_{\mathrm{p}}\right)$ respectively. The influence of this large number of transverse field sweeps on the remanent magnetization of the sample is examined. The experimental results are presented in Fig. 7(a), which shows the normalized magnetization $\mathrm{M}_{\mathrm{c}}$ measured by PPMS at the each moment $H_{\mathrm{ab}}=H_{\max }$. Successive cycles cause the magnetization to decrease by smaller and smaller amounts, as illustrated by the log-log plot of the data. The striking feature of Fig. 7 is that the induction $M_{\mathrm{c}}$ does not appear to saturate, even after a large number of field sweeps. Indeed, the $M_{\mathrm{c}}$ vs. cycle number $\mathrm{N}$ curves can be fitted using a power law $M_{\mathrm{c}} \sim \mathrm{N}^{-\alpha}$, as a straight line in log-log plot, with the exponent $\alpha$ being an increasing function of the transverse field amplitude $H_{\max }$.

Similar experimental conditions were used in the model, and the results are shown in Fig. 7(b). Good agreement between the experimental data and a power law fit is observed over the whole 


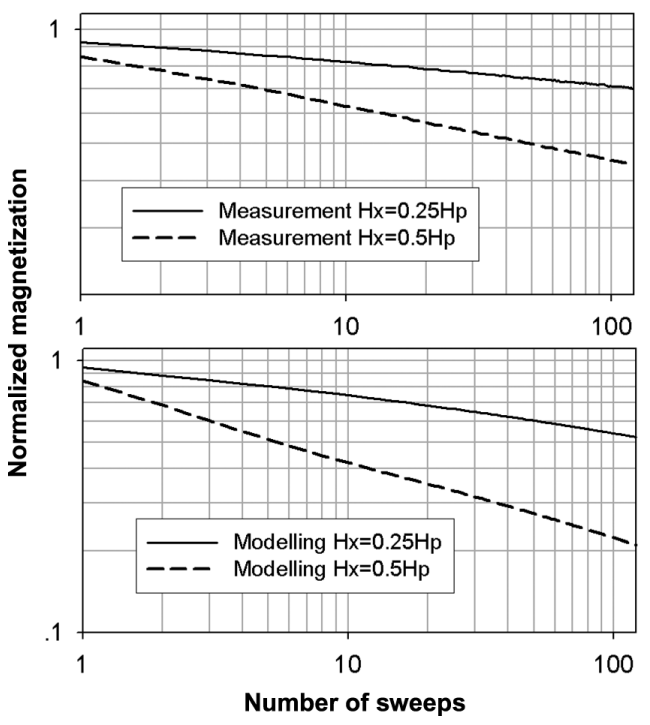

Fig. 7. Log-log plot of the measured and modeled magnetization at each peak positive transverse field for two different transverse field sweep amplitudes.

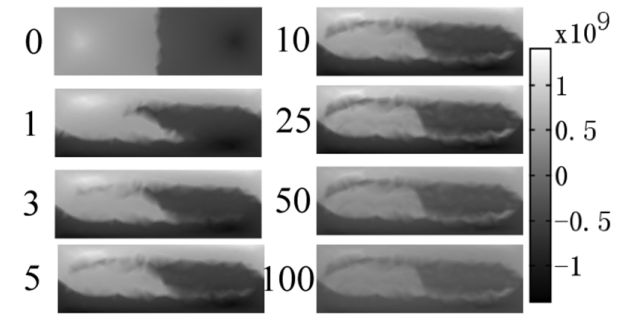

Fig. 8. Current distributions on the cross section of the sample at the end of the corresponding number of sweeps.

range of $\mathrm{N}(1<\mathrm{N}<100)$. As is observed experimentally, the remanent induction continues to decrease after 100 sweeps, and no saturation occurs.

The evolution of the current distribution over 100 sweeps is calculated by the model and shown in Fig. 8. The images are the current distribution on the cross section of the sample at the moment of the end of the 1st, 3rd, 5th, 10th, 25th, 50th and 100th sweep. As can be seen from the images, the current profile changes during the initial sweeps and reaches a relatively balanced distribution at the 10th sweep. These current profiles are made up of two regimes: regime 1 is two horizontal layers carrying positive and negative current located at the top and the bottom of the sample; regime 2 is the central area in which the original current distribution remains. The horizontal layers on the top and bottom no longer contribute to the c-axis magnetization. The forming of this current distribution gives the decay of $M_{\mathrm{c}}$ in the initial a few sweeps. After the 10th sweep, the magnitude of the current changes rather than the distribution.

There are two characteristic changes to the magnitude of the current; the first one is that the value of the current in regime 1 increases with a few sweeps (images 1-5) and decrease afterwards (images 10-100); the other is the magnitude of the current in the central region (regime 2) decreases. A reasonable explanation of this phenomenon is that the applied transverse field always tries to destroy the initially trapped magnetization, either by taking over the space which is originally occupied by the current contributed to $c$-axis magnetization (regime 2), or by reducing the magnitude of that current. However the transverse field would not affect the magnitude of the current in the top and bottom layers (regime 1) which contribute to $a b$-axis magnetization. In the mean while, $J_{\mathrm{c}}(\mathrm{B})$ increases as the local magnetic flux density decreases, that is the reason of the increase of the magnitude of the current density in regime 1 . The exponential decay of magnetization against the number of sweeps is determined mainly by the decrease of the magnitude of current density in regime 2 .

An important argument which must be clarified is whether this exponential decay of magnetization is due to the repeated application of the transverse field or just the normal flux creep relaxation. The contribution of the decay arising from flux-creep effects during a crossed field experiment can be estimated by the model. To be consistent with the experiment, the present model allows a time of $300 \mathrm{~s}$ to elapse after the magnetization process. The magnetization decreases by approximately $29 \%$ during this period and then by a further $0.13 \%$ during the application of one full cycle of the transverse field. Considering the period for a large number (100) of transverse field sweeps, the corresponding decrease due to flux creep is $4.5 \%$. Such values are much smaller then the decay in magnetization observed in Fig. 7(a) in which the magnetizations decrease for $30 \%$ and $55 \%$ for the two curves at the end of the 100th sweep. The discussion above shows that the decay of magnetization in Fig. 7 is mainly due to the crossed field effect.

\section{CONCLUSION}

The study in this paper focuses on the magnetization decay in a superconductor pre-magnetized along its shortest direction (c-axis) and then subjected to a series of transverse field cycles. The model shows that the suppression of magnetization results primarily from the modification of the current distribution in layers of the sample that are perpendicular to the direction of the pre-magnetization. The results obtained in the present study also allow significant conclusions to be drawn that a large number of sweeps in transverse field causes the magnetic induction to decrease continuously following a power law whose exponent is related to the sweep amplitude. The model proposed in this study offers a simple way of predicting the electromagnetic behavior of YBCO pellets subjected to crossed magnetic fields.

\section{REFERENCES}

[1] A. M. Campbell and D. A. Cardwell, "Bulk high temperature superconductors for magnet applications," Cryogenics, vol. 37, 1997.

[2] T. A. Coombs, A. M. Campbell, R. Storey, and R. Weller, "Superconducting magnetic bearings for energy storage flywheels," IEEE Trans. Appl. Supercond., vol. 9, 1999.

[3] P. Vanderbemden, Z. Hong, T. A. Coombs, S. Denis, M. Ausloos, J. Schwartz, I. B. Rutel, N. H. Babu, D. A. Cardwell, and A. M. Campbell, "Behavior of bulk high-temperature superconductors of finite thickness subjected to crossed magnetic fields: Experiment and model," Physical Review B, vol. 75, p. 174515, 2007.

[4] L. M. Fisher et al., "Superposition of currents in hard superconductors placed into crossed ac and dc magnetic fields," Solid State Commun., vol. 97, 1996.

[5] L. M. Fisher et al., "Collapse of the magnetic moment in a hard superconductor under the action of a transverse ac magnetic field," Physica C, vol. 278, 1997.

[6] D. A. Cardwell et al., "Round robin measurements of the flux trapping properties of melt processed $\mathrm{Sm}-\mathrm{Ba}-\mathrm{Cu}-\mathrm{O}$ bulk superconductors," Physica C, vol. 623, 2004.

[7] P. W. Anderson and Y. B. Kim, "Hard Superconductivity: Theory of the motion of Abrikosov flux lines," Review of Modern Physics, vol. 129, pp. 528-536, 1964. 